Article

\title{
Serum MicroRNAs as Biomarkers in Hepatitis C: Preliminary Evidence of a MicroRNA Panel for the Diagnosis of Hepatocellular Carcinoma
}

\author{
Anna Weis ${ }^{1,2}$, Louise Marquart ${ }^{3}$, Diego A. Calvopina ${ }^{1,2}$, Berit Genz ${ }^{1,2}$, Grant A. Ramm ${ }^{1,2, *, \dagger}$ \\ and Richard Skoien $1,2,4,+$ \\ 1 Hepatic Fibrosis Group, QIMR Berghofer Medical Research Institute, 300 Herston Rd, \\ Herston, QLD 4006, Australia; anna.weis1@uqconnect.edu.au (A.W.); \\ Diego.Calvopina@qimrberghofer.edu.au (D.A.C.); Berit.Genz@qimrberghofer.edu.au (B.G.); \\ Richard.Skoien@health.qld.gov.au (R.S.) \\ 2 Faculty of Medicine, University of Queensland, Herston Road, Herston, QLD 4006, Australia \\ 3 QIMR Berghofer Statistics Unit, QIMR Berghofer Medical Research Institute, 300 Herston Rd, \\ Herston, QLD 4006, Australia; Louise.Marquart@qimrberghofer.edu.au \\ 4 Department of Gastroenterology and Hepatology, Royal Brisbane and Women's Hospital, Bowen Bridge Rd \\ \& Butterfield St, Herston, QLD 4029, Australia \\ * Correspondence: Grant.Ramm@qimrberghofer.edu.au; Tel.: +61-7-3362-0177 \\ $\dagger$ These authors contributed equally to this work.
}

Received: 3 January 2019; Accepted: 14 February 2019; Published: 17 February 2019

Abstract: Early diagnosis of cirrhosis and hepatocellular carcinoma (HCC) due to chronic Hepatitis $\mathrm{C}(\mathrm{CHC})$ remain clinical priorities. In this pilot study, we assessed serum microRNA (miRNA) expression to distinguish cirrhosis and HCC, alone and in combination with the aminotransferase-toplatelet ratio (APRI), Fibrosis 4 (FIB-4), and alpha-fetoprotein (AFP). Sixty CHC patients were subdivided into 3 cohorts: Mild disease (fibrosis stage F0-2; $n=20$ ); cirrhosis $(n=20)$; and cirrhosis with HCC $(n=20)$. Circulating miRNA signatures were determined using a liver-specific real-time quantitative reverse transcription PCR (qRT-PCR) microarray assessing 372 miRNAs simultaneously. Differentially-expressed miRNA candidates were independently validated using qRT-PCR. Serum miRNA-409-3p was increased in cirrhosis versus mild disease. In HCC versus cirrhosis, miRNA-486-5p was increased, whereas miRNA-122-5p and miRNA-151a-5p were decreased. A logistic regression model-generated panel, consisting of miRNA-122-5p + miRNA-409-3p, distinguished cirrhosis from mild disease (area under the curve, AUC $=0.80$; sensitivity $=85 \%$, specificity $=70 \% ; p<0.001)$. When combined with FIB-4 or APRI, performance was improved with AUC $=0.89(p<0.001)$ and $0.87(p<0.001)$, respectively. A panel consisting of miRNA-122-5p + miRNA-486-5p + miRNA-142-3p distinguished HCC from cirrhosis $($ AUC $=0.94$; sensitivity = $80 \%$, specificity $=95 \% ; p<0.001)$, outperforming AFP (AUC $=0.64, p=0.065)$. Serum miRNAs are differentially expressed across the spectrum of disease severity in $\mathrm{CHC}$. MicroRNAs have great potential as diagnostic biomarkers in CHC, particularly in HCC where they outperform the only currently-used biomarker, AFP.

Keywords: serum miRNA; hepatocellular carcinoma; cirrhosis; non-invasive biomarker; hepatitis C

\section{Introduction}

Chronic Hepatitis $\mathrm{C}(\mathrm{CHC})$ is a major risk factor for the development of cirrhosis and subsequent hepatocellular carcinoma (HCC), a cancer whose incidence and mortality are increasing [1]. The impact of new, highly efficacious antiviral therapies [2] on morbidity and mortality largely depends 
on improved identification of cirrhosis due to $\mathrm{CHC}$ and its complications, including HCC. Despite treatment, the burden of CHC-related HCC in most countries is increasing and is expected to peak in the next decade [3]. Furthermore, whilst achieving a sustained virologic response (SVR) has been shown to improve liver function in cirrhotic patients, the risk of complications, such as HCC, remains [4]. There remain clinical imperatives to accurately identify patients with cirrhosis and improve diagnostic tests for CHC-related HCC.

The gold standard for the identification of cirrhosis for the purposes of HCC surveillance is liver biopsy, but this is invasive, prone to sampling error, and does not reflect the clinical spectrum of cirrhosis and is therefore now rarely used. Non-invasive tools, such as liver stiffness assessment (e.g., transient elastography), have been embraced to stage fibrosis in CHC patients, but a clinically-significant "indeterminate" range affecting the test accuracy exists [5]. Non-invasive biomarker panels, such as the aspartate aminotransferase to platelet ratio (APRI) [6] and Fibrosis-4 (FIB-4) [7] scores, provide good sensitivity and specificity within defined cut-offs, but values falling short of these specific thresholds lack diagnostic accuracy. Access to non-invasive tools is also sometimes limited to specialist treatment, pathology, or imaging centres.

The only currently-accepted HCC tumour biomarker, alpha-fetoprotein (AFP), generally has suboptimal sensitivity and specificity for surveillance and diagnosis in most cases [8]. Routine HCC surveillance comprises 6-monthly ultrasounds, with dynamic scanning (CT/MRI) used to further characterise suspicious lesions. For a proportion of tumours, however, diagnosis is necessarily delayed until interval growth can be demonstrated, limiting early access to definitive treatment. Indeed, although the sensitivity and specificity of ultrasound-based diagnostic strategies exceed $90 \%$ for the detection of HCC across all stages, its sensitivity is only $63 \%$ for early HCC [8].

Novel biomarkers, such as microRNAs (miRNAs), are being investigated for their diagnostic potential in viral hepatitis, hepatic fibrosis, and HCC. miRNAs are small non-coding RNAs involved in the regulation of gene expression at a post-transcriptional level. The stability of miRNAs in the circulation, as well as both healthy and diseased tissue, make them ideal potential biomarkers [9]. Preliminary studies of miRNAs in CHC-related HCC have identified promising candidates $[10,11]$, with the development of miRNA panels now being explored to improve diagnostic accuracy.

In this pilot study, we sought to identify differentially-expressed serum miRNAs in CHC patients with and without cirrhosis, as well as those with CHC-related HCC. We also sought to develop miRNA panels of the most promising diagnostic candidates, alone or in combination with APRI, FIB-4, or AFP, to explore their potential as novel non-invasive diagnostic tests.

\section{Results}

\subsection{Patient Characteristics}

The patient demographics and clinical characteristics of the $\mathrm{CHC}$ patient cohort are summarised in Table S2. All patients with mild disease (F0-2) and cirrhosis (F4) were followed for a mean of 16.7 and 21.2 months from sample collection, respectively, without any evidence of clinical decompensation or HCC development. There were no significant differences in CHC genotypes or treatment history between the three patient cohorts $(p>0.05)$.

\subsection{Differential miRNA Expression in Chronic Hepatitis $\mathrm{C}$ (CHC)}

Seven candidate miRNAs with 2-fold or more differential expression in F0-2 vs. F4 vs. HCC on the microarray were identified for further validation: miRNA-19b-3p, miRNA-22-3p, miRNA-122-5p, miRNA-142-3p, miRNA-151a-5p, miRNA-409-3p, and miRNA-486-5p. Differential expression levels on qRT-PCR of the selected miRNA candidates are presented in Figure 1. miRNA-409-3p was significantly upregulated in cirrhosis compared with mild disease ( $p=0.023$; Table 1 and Figure 1).

In HCC, serum levels of miRNA-122-5p and miRNA-151a-5p were significantly decreased $(p<0.001$ and $p=0.039$, respectively), whereas expression of miRNA-486-5p was significantly increased 
in HCC patients ( $p=0.001$ ), compared with F4 patients (Table 1, Figure 1). Expression of miRNA-142-3p was significantly decreased, while miRNA-19b-3p was significantly increased, in HCC compared with mild disease ( $p=0.039$ and $p=0.015$, respectively; Table 1, Figure 1). Significant differential expression of miRNA-22-3p was not confirmed on qRT-PCR, with only a trend towards increased expression in cirrhosis vs. mild disease $(p=0.056)$. Using receiver operating characteristic (ROC) curves, area under the curve (AUC) analysis was performed to assess the potential of individual candidate miRNAs to discriminate cirrhosis (from mild disease) and HCC (from cirrhosis alone), with fair performance only (AUCs 0.60-0.85; Figure 2).



(C) microRNA-151a-5p

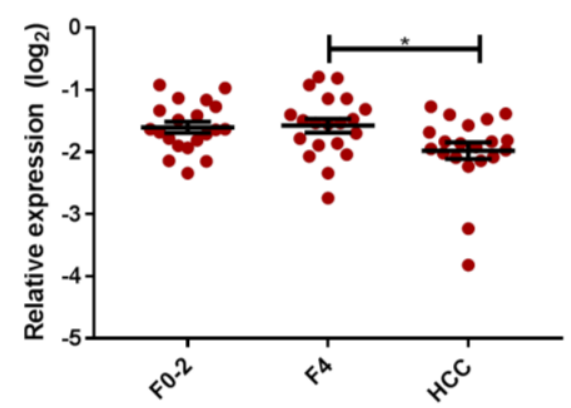

(E) microRNA-409-3p





(D) microRNA-142-3p
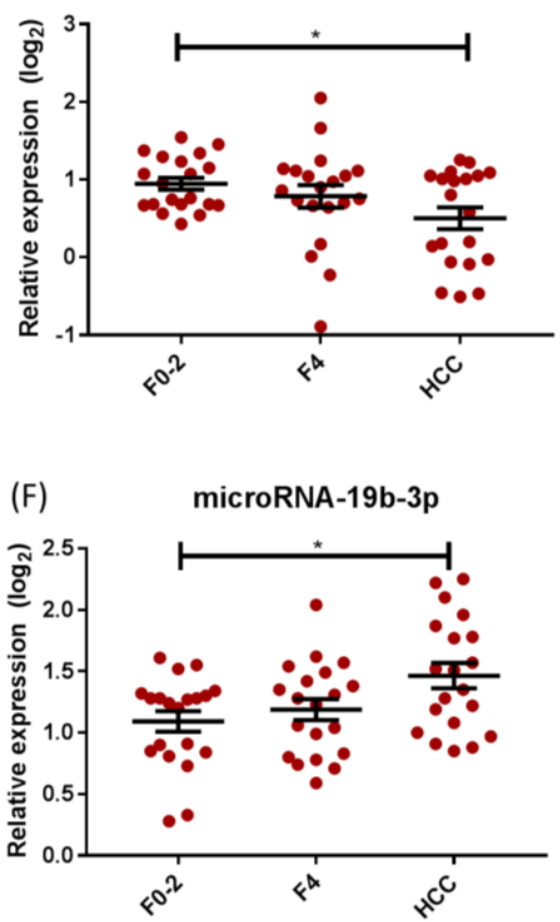

Figure 1. Association between circulating miRNA levels and severity of hepatic fibrosis and HCC in CHC. (A) miRNA-122-5p, (C) miRNA-151a-5p, and (D) miRNA-142-3p show a decreased expression in HCC, compared to cirrhosis, whereas (B) miRNA-486-5p shows an increased expression in HCC, compared to cirrhosis. (E) miRNA-409-3p shows an increased expression in F4, when compared to mild disease. (F) miRNA-19b-3p shows an increased expression in HCC compared to mild disease. Lines represent mean expression levels $( \pm \mathrm{SEM}) .{ }^{*} p<0.05,{ }^{* *} p<0.01,{ }^{* * *} p<0.001$. (Abbreviations: $\mathrm{CHC}=$ chronic Hepatitis C; F0-2 = mild disease; F4 = cirrhosis; HCC = hepatocellular carcinoma; miRNA = microRNA; SEM = standard error of the mean). 
Table 1. Associations of circulating miRNA expression with the severity of disease in chronic Hepatitis C: Validation of selected miRNA expression levels using qRT-PCR.

\begin{tabular}{ccccc}
\hline \multirow{2}{*}{ miRNA } & \multirow{2}{*}{ Overall $\boldsymbol{p}$-Value (ANOVA) } & \multicolumn{3}{c}{$\boldsymbol{p}$-Value } \\
\cline { 3 - 5 } & & F0-2 vs. F4 & F0-2 vs. HCC & F4 vs. HCC \\
\hline miRNA-122-5p & $<0.001$ & 0.066 & 0.103 & $<0.001$ \\
miRNA-486-5p & $<0.001$ & 0.999 & 0.001 & 0.001 \\
miRNA-151a-5p & 0.025 & 0.986 & 0.056 & 0.039 \\
miRNA-409-3p & 0.030 & 0.023 & 0.417 & 0.320 \\
miRNA-19b-3p & 0.015 & 0.734 & 0.015 & 0.089 \\
miRNA-142-3p & 0.047 & 0.636 & 0.039 & 0.253 \\
\hline
\end{tabular}

$p$-values were calculated using the $2^{\Delta \mathrm{Ct}}$ normalisation method and ANOVA with Tukey's post hoc test with significance designated by a $p$-value $<0.05$. (Abbreviations: ANOVA $=$ analysis of variance; F0- $2=$ mild disease; F4 = cirrhosis; HCC = cirrhosis with hepatocellular carcinoma; miRNA = microRNA; qRT-PCR = quantitative real time reverse transcription PCR).

(A)

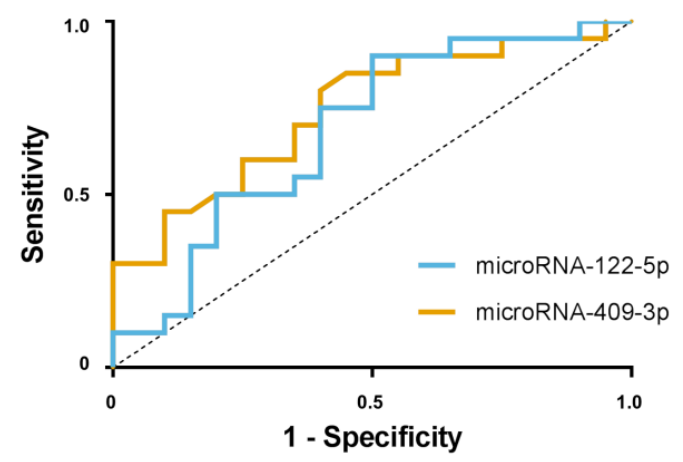

(B)

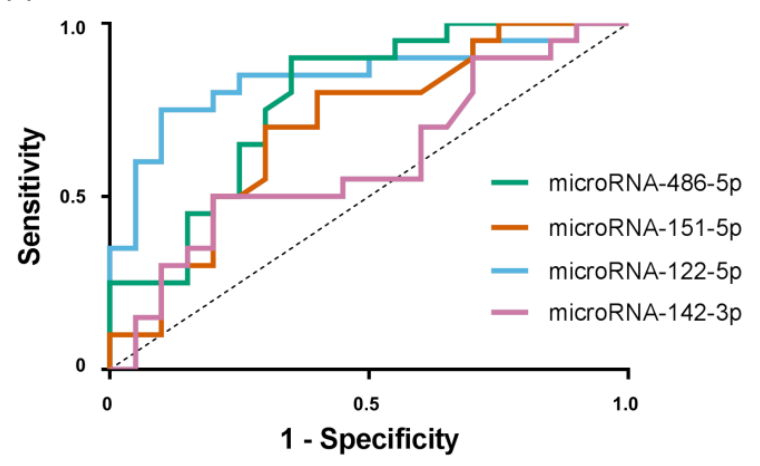

Figure 2. ROC curves for individual serum miRNAs to identify cirrhosis or HCC in chronic hepatitis C (A). ROC curve performance of miRNA-122-5p with AUC of 0.69 (95\% CI 0.51-0.86, $p=0.023$ ) and miRNA-409-3p with AUC of 0.74 (95\% CI 0.58-0.90, $p=0.004)$ to identify cirrhosis when compared to mild disease. (B) ROC curve performance of selected miRNAs to identify HCC when compared to cirrhosis as follows: miRNA-486-5p, AUC of 0.78 (95\% CI 0.63-0.93, $p<0.001$ ); miRNA-151a-5p, AUC of 0.70 (95\% CI 0.54-0.87, $p=0.014$ ); miRNA-122-5p, AUC of 0.85 (95\% CI 0.72-0.97, $p<0.001$ ); miRNA-142-3p, AUC of 0.60 (95\% CI 0.42-0.78, $p=0.140$ ). (Abbreviations: AUC = area under the curve; HCC = hepatocellular carcinoma; miRNA = microRNA; ROC = receiver operating characteristic; $95 \% \mathrm{CI}=95 \%$ confidence interval).

\subsection{Discriminating Cirrhosis and Hepatocellular Carcinoma (HCC) Using Serum miRNA Panels}

We used stepwise logistic regression, using forward selection and backward elimination, to develop serum miRNA panels that could distinguish cirrhosis or HCC (from mild disease and cirrhosis, respectively). A panel comprising miRNA-122-5p and miRNA-409-3p demonstrated optimal differential diagnostic performance for cirrhosis (vs. F0-2) with an AUC of 0.80 (95\% CI $0.66-0.95, p<0.001$ ), sensitivity of $85 \%$, specificity of $70 \%$, negative predictive value (NPV) of $82 \%$, positive predictive value (PPV) of 74\%, and overall accuracy of 78\% (Figure 3A). For HCC (vs. F4), an optimal panel comprising miRNA-122-5p, miRNA-486-5p, and miRNA-142-3p demonstrated excellent diagnostic performance with an AUC of 0.94 (95\% CI 0.87-1.00, $p<0.001$ ), sensitivity of $80 \%$, specificity of $95 \%$, NPV of $94 \%$, PPV of $83 \%$, and overall accuracy of $88 \%$ (Figure 3B).

\subsection{Cross Validation of Serum miRNA Panels}

To further assess the robustness of the miRNA panel diagnostic performance, k-fold cross validation was performed using 5-fold cross-validation. Our cirrhosis miRNA panel, combining miRNA-409-3p and miRNA-122-5p, resulted in an AUC of 0.79 (95\% CI 0.65-0.93), with a sensitivity of $85 \%$, specificity of $65 \%$, NPV of $76 \%$, PPV of $75 \%$, and overall accuracy of $78 \%$ (Figure 4 A). Our HCC 
miRNA panel, combining miRNA-122-5p, miRNA-486-5p, and miRNA-142-3p, resulted in an AUC of 0.91 (95\% CI $0.81-1.0)$, sensitivity of $81 \%$, specificity of $87 \%$, NPV of $80 \%$, PPV of $91 \%$, and overall accuracy of $85 \%$ (Figure $4 \mathrm{~B}$ ).
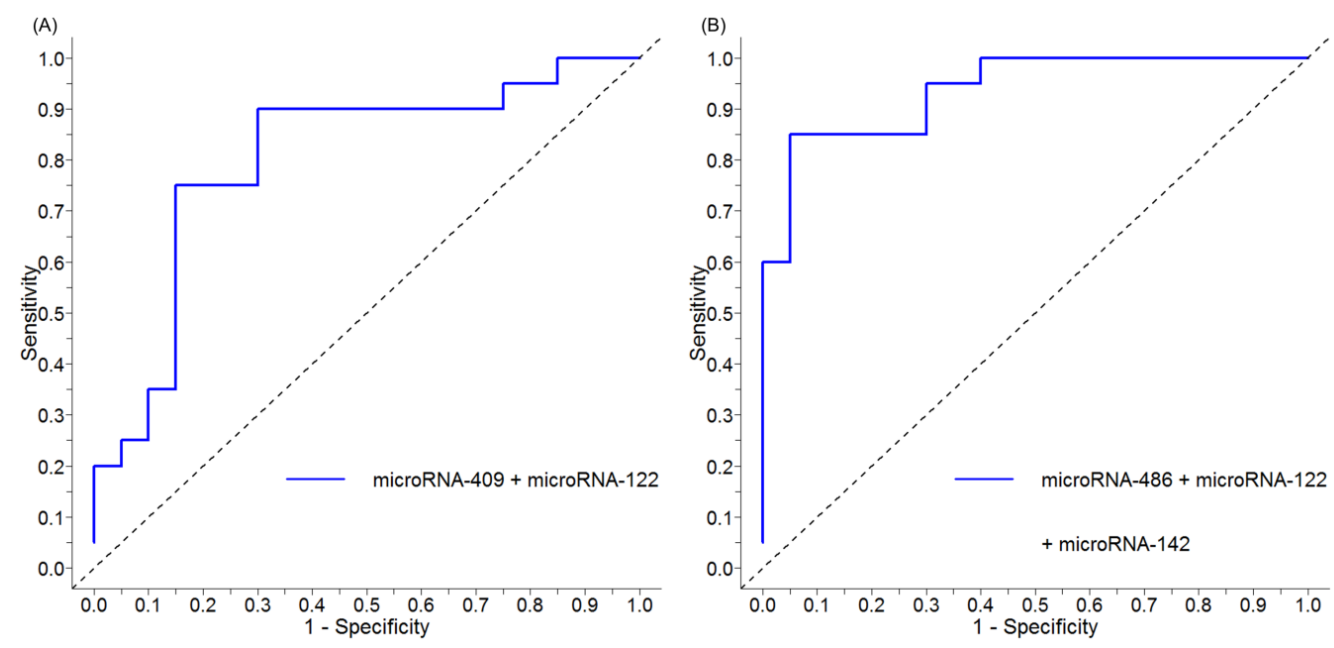

Figure 3. ROC curve of serum miRNA panels to identify cirrhosis or HCC in chronic Hepatitis C (A) miRNA panel (miRNA-122-5p and miRNA-409-3p) performance to identify cirrhosis, compared to mild disease, with AUC of 0.80 (95\% CI 0.66-0.95, $p<0.001)$. (B) miRNA panel to identify HCC, compared to cirrhosis, using miRNA-122-5p, miRNA-486-5p, and miRNA-142-3p with AUC of 0.94 (95\% CI 0.87-1.00, $p<0.001$ ). (Abbreviations: AUC = area under the curve; F0-2 = mild disease; F4 = cirrhosis; $\mathrm{HCC}=$ hepatocellular carcinoma; miRNA = microRNA; ROC = receiver operating characteristic; $95 \% \mathrm{CI}$ $=95 \%$ confidence interval).
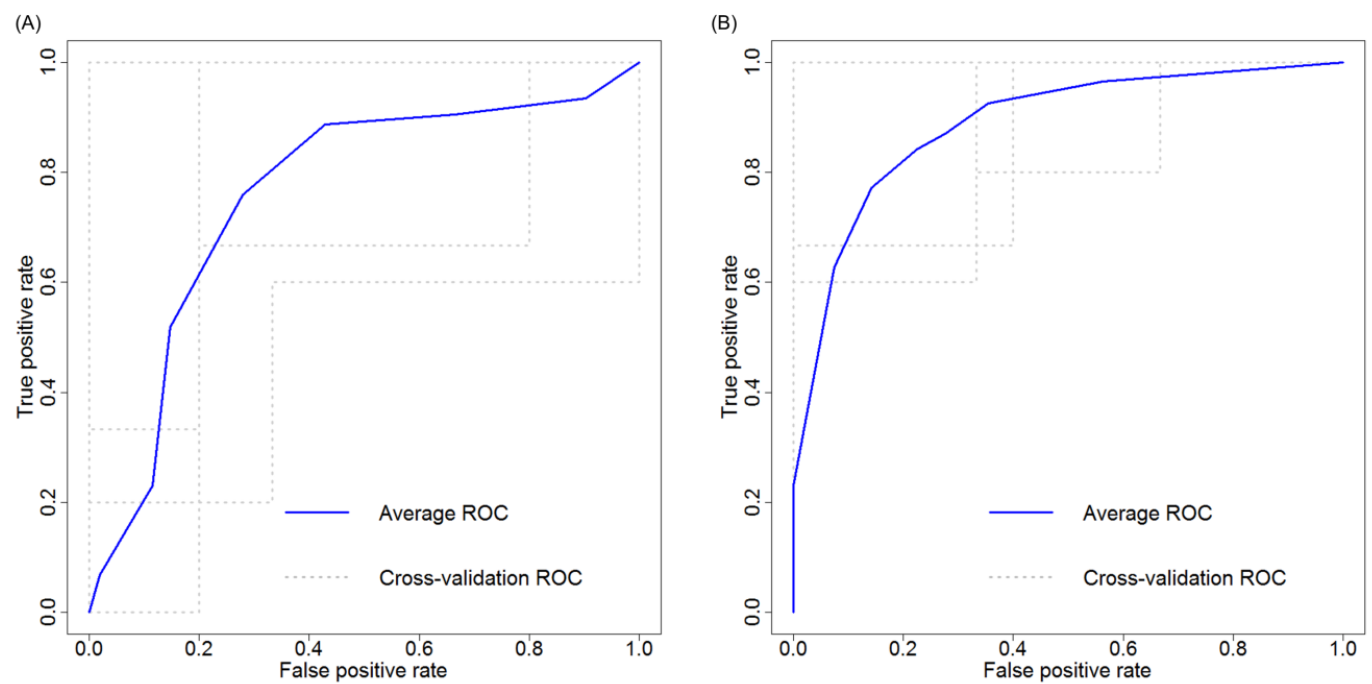

Figure 4. K-fold (5-fold) cross validation ROC curve of serum miRNA panels to identify cirrhosis or HCC in chronic Hepatitis C (A) miRNA panel (miRNA-122-5p and miRNA-409-3p) 5-fold cross validation performance to identify cirrhosis, compared to mild disease, demonstrates an AUC of 0.79 (95\% CI 0.65-0.93). (B) miRNA panel to identify HCC, compared to cirrhosis, using miRNA-122-5p, miRNA-486-5p, and miRNA-142-3p, demonstrates an AUC of 0.91 (95\% CI 0.81-1.0). (Abbreviations: AUC = area under the curve; F0-2 = mild disease; F4 = cirrhosis; $\mathrm{HCC}=$ hepatocellular carcinoma; miRNA $=$ microRNA; ROC $=$ receiver operating characteristic; $95 \% \mathrm{CI}=95 \%$ confidence interval). 
2.5. Comparative Performance of the Aspartate Aminotransferase-to-Platelet Ratio (APRI) and Fibrosis 4 $($ FIB-4) \pm Serum miRNA Panel to Discriminate Cirrhosis

The diagnostic performance of the biochemical indices, FIB-4 and APRI, to discriminate cirrhosis (F4) from mild disease (F0-2) in our cohort was also assessed. Using the currently accepted FIB-4 cut-off of 1.45 (to exclude advanced fibrosis) resulted in an AUC of 0.87 (95\% CI 0.75-0.98, $p<0.001$ ) with a sensitivity of $95 \%$, specificity of $50 \%$, NPV of $92 \%$, PPV of $70 \%$, and overall accuracy of $78 \%$ (Figure 5A). Applying the commonly accepted APRI cut-off of 1.0 (to exclude cirrhosis) resulted in an AUC of 0.84 (95\% CI $0.71-0.97, p<0.001)$, with a sensitivity of $80 \%$, specificity of $75 \%$, NPV of $79 \%$, PPV of $76 \%$, and overall accuracy of $78 \%$ (Figure $5 \mathrm{~B}$ ). Combining these scores with our cirrhosis miRNA panel marginally improved the diagnostic performance with AUCs of 0.87 and 0.89 for APRI and FIB-4, respectively.
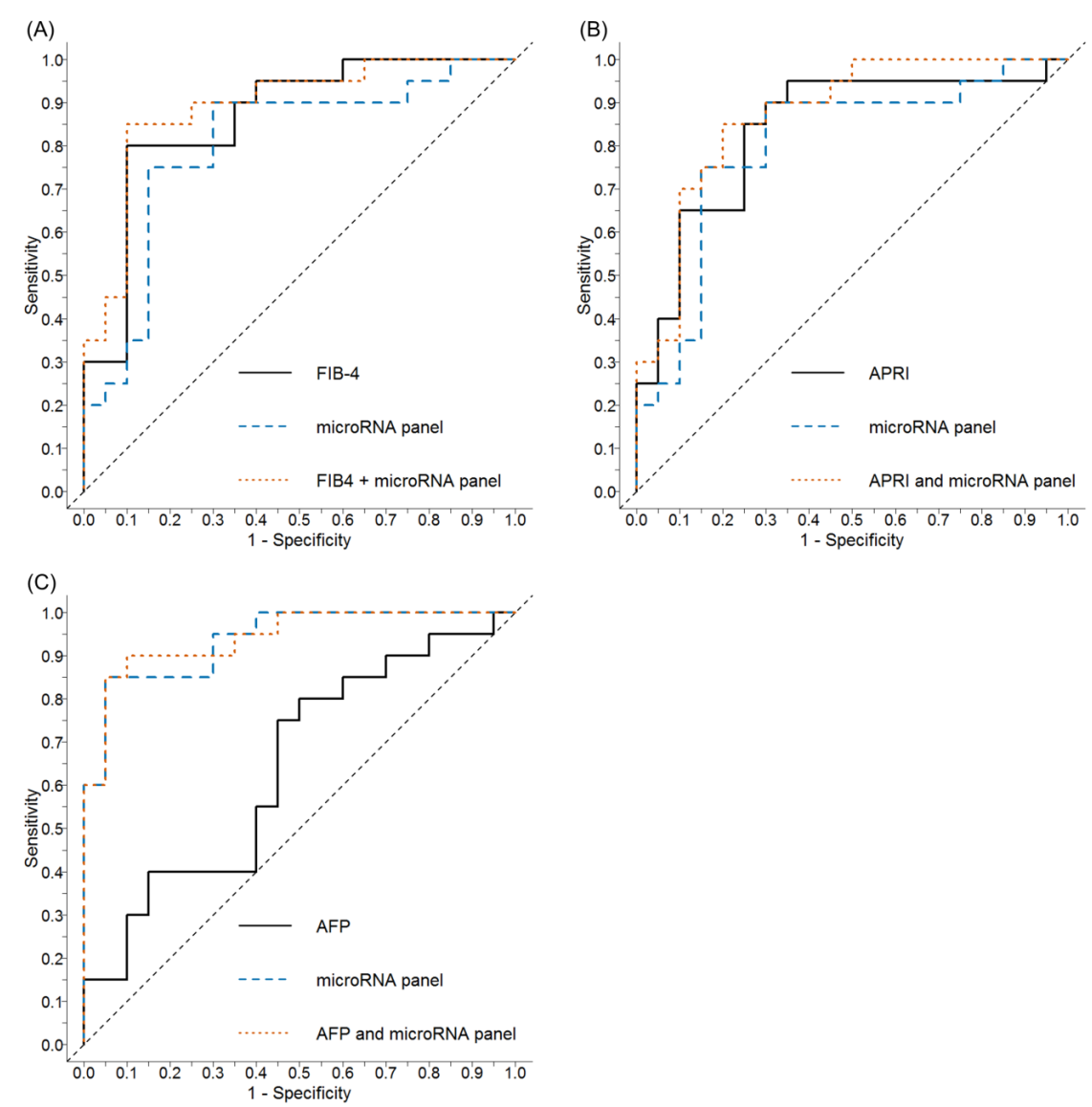

Figure 5. ROC curves to identify cirrhosis or HCC using FIB-4 or APRI alone, or in combination with circulating miRNA panels in chronic Hepatitis C. (A) In distinguishing cirrhosis, the performance of FIB-4 (AUC $=0.87,95 \%$ CI 0.75-0.98, $p<0.001$; using a cut-off of $>1.45$ to exclude advanced fibrosis) was enhanced in combination with the cirrhosis miRNA panel described in Figure 3A (AUC $=0.89,95 \%$ CI $0.79-0.99, p<0.001$ ); (B) in distinguishing cirrhosis, the performance of APRI (AUC $=0.84,95 \%$ CI $0.71-0.97, p<0.001$; using a cut-off of $>1.0$ to exclude cirrhosis) was enhanced in combination with the cirrhosis miRNA panel described in Figure 3A (AUC $=0.87,95 \%$ CI 0.76-0.98, $p<0.001$ ); (C) AFP alone demonstrated poor diagnostic utility in the detection of HCC (AUC $=0.64,95 \%$ CI $0.47-0.82$, $p=0.065$; using a cut-off of $>20$ to diagnose HCC) and when used in combination with the HCC miRNA panel described in Figure 3B there was no improvement in the HCC miRNA panel's ability to detect HCC (AUC $=0.94,95 \%$ CI 0.88-1.00, $p<0.001$ ). (Abbreviations: APRI = aspartate aminotransferase to platelet ratio; $\mathrm{AFP}=$ alpha-fetoprotein; $\mathrm{AUC}=$ area under the curve; FIB- $4=$ Fibrosis -4 biomarker panel; $\mathrm{HCC}=$ hepatocellular carcinoma; miRNA = microRNA; ROC = receiver operating characteristic; $95 \% \mathrm{CI}=95 \%$ confidence interval). 


\subsection{Comparative Performance of Alpha-Fetoprotein $(A F P) \pm$ Serum miRNA Panel to Discriminate HCC}

In this cohort, the currently accepted tumour marker, AFP, was a poor discriminator of HCC with an AUC of 0.64 (95\% CI 0.47-0.82, $p=0.065)$. Using a commonly accepted AFP cut-off of $>20 \mu \mathrm{g} / \mathrm{L}$ to identify HCC produced a sensitivity of $25 \%$, specificity of $90 \%$, NPV of $55 \%$, PPV of $71 \%$, and overall accuracy of $58 \%$. Combining AFP with our HCC miRNA panel did not improve the diagnostic performance with an AUC of 0.94 (95\% CI 0.88-1.00, $p<0.001$; Figure 5C).

\section{Discussion}

Despite the availability of new, highly-efficacious treatments, $\mathrm{CHC}$ remains a huge public health burden with millions of infected patients affected by cirrhosis worldwide [3]. Distinguishing cirrhosis from mild disease remains essential as such patients warrant ongoing surveillance for complications, such as HCC, even after SVR. The invasive nature of the gold standard liver biopsy and the absence of highly accurate non-invasive biomarkers remain important challenges in routine patient care [12]. Although miRNAs demonstrate potential as biomarkers, with the identification of several candidate miRNAs in many diseases (including HCC), optimal diagnostic panels have not been developed.

In this pilot study, we used serum samples from 60 well-characterised $\mathrm{CHC}$ patients across the spectrum of disease to systematically identify the most promising potential miRNA biomarkers. During the discovery phase, differentially-expressed miRNAs were identified via liver-specific microarray. The most promising candidates were then validated using independent qRT-PCR and then optimised as miRNA panels to test their diagnostic accuracy compared with currently-accepted non-invasive clinical tools. Our data strengthen the case for further exploration of serum miRNAs as clinically-useful biomarkers in cirrhosis and HCC.

The circulating serum level of miRNA-409-3p was significantly increased in cirrhotic patients (F4) compared to those with only mild disease (F0-2). There is little in the literature describing the role of miRNA-409-3p in fibrosis progression in liver disease, although increased plasma expression levels correlated with markers of liver injury (gamma-glutamyl-transferase, alkaline phosphatase) in a murine neoplastic model of hereditary tyrosinemia type 1 [13]. As a cancer biomarker, studies using in vitro models of prostate cancer have reported increased miRNA-409-3p levels [14]. Interestingly, this was in association with epithelial to mesenchymal transition, a purported mechanism of fibrosis progression in liver disease. Reduced expression of miRNA-409-3p in other cancers, such as breast cancer [15], suggests a tissue-specific role for miRNA-409-3p.

miRNA-122-5p is the most abundant miRNA expressed in the liver [16]. In CHC, circulating miRNA-122-5p levels have been shown to correlate with serum ALT and AST levels and tissue necrosis and inflammation [11,17]. In the current study, we demonstrated that circulating serum levels of miRNA-122-5p were significantly decreased in CHC patients with HCC compared with cirrhosis alone. A previous study reported no significant differences in serum miRNA-122-5p levels in HCC patients [18], but this study included patients with multiple disease aetiologies rather than CHC patients alone. Conversely, increased HCC tissue expression levels have been described for miRNA-122-5p, including CHC-related HCC $[19,20]$. Differential peripheral versus tissue expression is commonly-described with miRNAs in cancer, highlighting their potential application as clinical biomarkers, although specific expression profiles are yet to be definitively established. For example, reduced tissue expression in HCC has been shown to correlate with poor prognosis and metastasis [19], while poorer overall survival has also been described in HCC patients with lower levels of circulating miRNA-122-5p [18]. While the precise mechanisms involved require further investigation, these data clearly suggest the clinical importance of miRNA-122-5p in HCC and the current study provides consistent evidence that miRNA-122-5p may have utility as a non-invasive serum biomarker in HCC.

In the current study, circulating miRNA-486-5p was the only miRNA with an increased peripheral expression in CHC-related HCC compared with cirrhosis alone. Higher levels of circulating miRNA-486-5p have been previously described in patients with HCC compared with healthy controls, although the aetiology of HCC and cirrhosis status was not reported [21]. Higher serum expression of 
miRNA-486-5p has been associated with longer recurrence-free survival in HCC patients [22], while reduced expression in HCC tissue compared with adjacent non-tumorous tissue has been reported in multiple chronic liver disease aetiologies, including CHC-related HCC [23,24]. Expression levels also appear to correlate with earlier HCC recurrence following resection [23]. Downstream targets of miRNA-486-5p include Phosphoinositide-3-Kinase Regulatory Subunit 1 (PIK3R1) and Never in Mitosis gene a-(NIMA-)Related Kinase 2 (NEK2), which have been shown to play important roles in HCC proliferation, migration, and invasion [23,24]. Furthermore, miRNA-486-5p is located on chromosome 8q11.21, an area commonly deleted in HCC, which possibly accounts for reduced tissue expression in HCC and supports its potential role as a tumour suppressor [25]. Our finding of differential serum expression of miRNA-486-5p in HCC further promotes the importance and clinical potential of this miRNA.

The current study demonstrated significantly reduced expression of circulating miRNA-151a-5p in cirrhotic patients with HCC compared with cirrhosis alone. Others have described increased plasma expression of miRNA-151a-5p in viral hepatitis-induced HCC, but this was in comparison with healthy controls and non-viral hepatitis-related HCC patients [26]. Indeed, increased tissue expression levels of miRNA-151a-5p have been described in HCC tissue, compared with adjacent non-tumorous tissue [25,27], and miRNA-151a-5p is located on chromosome 8q24.3, which is an area that is frequently amplified in HCC $[25,27]$. High tissue expression levels of miRNA-151a-5p have been correlated with intrahepatic metastasis, cell migration, and invasion [25], while FAK (the focal adhesion kinase gene that is the host gene of miRNA-151a-5p) is usually co-expressed with miRNA-151a-5p, and it has been suggested that the two function synergistically to enhance HCC cell motility [25]. There is, therefore, growing evidence that miRNA-151a-5p may have a pro-oncogenic function, highlighting the clinical potential of identifying it as one of the differentially-expressed candidates in our cohort.

We performed ROC curve analysis using stepwise logistic regression to identify an optimal miRNA panel to distinguish cirrhosis from mild disease in CHC. The resulting cirrhosis miRNA panel (comprising miRNA-122-5p and miRNA-409-3p) resulted in an AUC of 0.80. Combining the panel with APRI or FIB-4 only marginally improved the performance of either score alone (AUCs of 0.87 and 0.89 , respectively), but illustrates the future clinical potential of combining miRNA expression with other non-invasive biomarkers to improve diagnostic accuracy. ROC curve analysis of our optimal HCC miRNA panel, comprising miRNA-122-5p, miRNA-486-5p, and miRNA-142-3p, demonstrated an excellent ability to distinguish patients with HCC from those with cirrhosis alone, with an AUC of 0.94 . This result was substantially better than the diagnostic performance of AFP to identify HCC (AUC of 0.64). It also statistically out-performed the more intuitive combination of miRNA-122-5p, miRNA-486-5p, and miRNA-151a-5p (AUC 0.91) due to co-linear expression of miRNA-122-5p and miRNA-151a-5p. Combining AFP with our miRNA panel did not improve its diagnostic performance. While prospective validation of our HCC miRNA panel in a larger cohort is warranted, these results clearly demonstrate the clinical potential of this serum miRNA panel, with improved sensitivity ( $80 \%$ vs. $25 \%$ ) and specificity ( $95 \%$ vs. $90 \%$ ) compared with the only HCC tumour biomarker routinely used in clinical practice (AFP).

This study contributes to the considerable growing interest in miRNAs as biomarkers of chronic liver disease in recent years. Meta-analyses $[28,29]$ have concluded that miRNAs have great potential as non-invasive biomarkers, especially in the diagnosis of HCC, and a number of potential miRNAs candidates have been identified. These analyses found, however, that the quality of studies in this area is highly variable, significant heterogeneity exists, and results are often contradictory. Indeed, the published data suggest that high frequency miRNAs (such as miRNA-122-5p in HCC) may ultimately be the most specific candidates, and combinations of non-invasive biomarkers and/or panels of miRNAs are now believed to be the most likely strategy in accurately distinguishing disease [29]. Our data are consistent with these conclusions. We identified that optimal diagnostic performance was achieved using combinations of significantly differentially-expressed miRNAs, including commonly-expressed miRNAs. Further high-quality prospective research is needed to 
validate these promising preliminary results. The current study also confirmed a number of candidate miRNAs that have been implicated in other HCC studies, including a novel approach that assessed circulating miRNA levels significantly associated with HCC in viral hepatitis in an attempt to establish a predictive model [30]. Such candidates could be further investigated in functional studies to identify downstream targets to elucidate their roles in disease pathogenesis and potentially contribute to the development of new therapeutic strategies.

There are some limitations to this study that need to be considered when evaluating our results. First, our study population only included two patients who had achieved an SVR and genotypes 1 and 3 were also over-represented in our cohort, as they are the most common genotypes in Australia. A clinically useful diagnostic miRNA panel would ideally demonstrate accuracy across all genotypes and also in cirrhotic patients following viral eradication; second, in the HCC cohort, serum samples were not obtained following definitive HCC treatment meaning dynamic changes of our proposed diagnostic serum miRNAs according to tumour viability could not be assessed. While this could lend additional weight to our results, this was not undertaken in the current study as treatments (and therefore outcomes) varied considerably amongst the cohort, thus making data difficult to interpret. Additionally, there was the risk of a type 2 error in the interpretation of any data due to the sample size in this pilot cohort; third, patients in this study were allocated to clinically-significant cohorts based on standard-of-care expert assessment instead of being formally staged with gold standard diagnostic biopsy, meaning discriminating between individual fibrosis stages based on miRNA expression was beyond the scope of this study. Clearly, a study using both current standard-of-care clinical assessment and liver biopsy would be desirable, but treatment of Hepatitis $C$ in the era of highly efficacious direct-acting antivirals rarely involves liver biopsy; finally, and perhaps most importantly, this was a pilot study with small numbers of (albeit well-characterised) patients that aimed to test hypotheses, generate preliminary data, and contribute to our understanding of this area. To address the robustness of our data, we performed an internal 5-fold validation, but we acknowledge the importance of an external, preferably prospective, validation study from investigators with sufficiently large patient cohorts.

Compared with recent studies, however, there are a number of important methodological strengths of the current study. First, we used clinically-relevant controls (i.e., mild disease that is definitely not cirrhosis vs. established cirrhosis; cirrhosis alone vs. cirrhosis complicated by HCC) instead of healthy controls. Additionally, as even gold standard liver biopsy is associated with fibrosis stage sampling errors, we suggest that the use of these clinically-relevant patient cohorts, rather than sub-dividing patients according to individual METAVIR stages, may provide more meaningful data to investigators; second, we measured circulating rather than tissue-based miRNA levels as peripheral samples are much more accessible to clinicians and a blood-based diagnostic biomarker has huge logistic advantages over a tissue-based one; finally, we compared expression levels in serum rather than plasma, which, as is increasingly accepted, deliver suboptimal results [29]. We suggest that the results of this pilot study, taken in the context of emerging data in this field, represent an important contribution to the literature and further highlight the roles of specific miRNAs as promising non-invasive biomarkers. The excellent performance of our miRNA panel, compared with AFP as the only clinically-accepted biomarker in HCC, also suggests that we may not be too far away from a validated diagnostic HCC miRNA panel that could be used in clinical practice.

\section{Materials and Methods}

\subsection{Patient Recruitment and Characteristics}

All study procedures were undertaken in accordance with organisational ethical standards on human experimentation with approval of the Human Research Ethics Committees of the Royal Brisbane and Women's Hospital (HREC/13/QRBW/308, approval 12/12/2013) and QIMR Berghofer Medical Research Institute (P1509, approval 07/12/2012), and in accordance with the Declaration of 
Helsinki 1975 (revised 2013). Informed consent was obtained from all patients. Sixty CHC patients were retrospectively subdivided into 3 cohorts as follows: Mild disease without advanced fibrosis (F0-2; $n=20)$; cirrhosis (F4; $n=20)$; and cirrhosis with HCC (HCC; $n=20)$. Detailed patient characteristics are described in Supplementary Methods and Supplementary Table S1.

\subsection{RNA Extractions and Reverse Transcription}

For the screening phase, RNA was extracted from serum using the miRNeasy Serum/Plasma Kit (Qiagen; Hilden, Germany) and reverse-transcribed using the miScript II RT Kit (Qiagen). During the validation phase, serum RNA was extracted using the Plasma/Serum RNA Purification Mini Kit (Norgen Biotek Corp; Thorold, ON, Canada) and reversed-transcribed using the miRCURY LNA ${ }^{\mathrm{TM}}$ universal RT miRNA PCR Kit (Exiqon, Vedbaek, Denmark). RNA extraction, protocol modifications, and reverse transcription are described in Supplementary Methods. Primer target sequences for candidate miRNAs are shown in Supplementary Table S2.

\section{3. miRNA PCR Array, qRT-PCR, and Data Analysis}

During the screening phase, a miRNA PCR Array (Human Liver miFinder miScript miRNA PCR Array MIHS-3116ZG; Qiagen) was used to simultaneously measure the expression of 372 liver-related miRNAs in all 60 samples. The most significant differentially-expressed miRNAs ( $>2$-fold change and $p<0.05$ ) were selected for further validation. Leading miRNA candidates were independently validated by qRT-PCR (miRCURY LNA ${ }^{\mathrm{TM}}$ miRNA kit, see Supplementary Methods).

Following the screening phase, miRNA array data were normalised using quantile normalisation and analysed for differentially-expressed miRNAs using pairwise comparison of groups of interest with the modified t-test (limma package, R). miRNA candidates were selected based on $p<0.05$. Validation qRT-PCR data were analysed using the $2^{\Delta C t}$ method and expression values normalized to let-7i-5p and miRNA-23a-3p. Significant differential expression was tested using one-way ANOVA with Tukey's post-hoc pairwise comparison, with $p<0.05$ used to define statistical significance. Data normalisation and statistical analysis were performed using GraphPad Prism 7 (GraphPad Software Inc; San Diego, CA, USA) and R (version 3.3.3, https: / / www.r-project.org) [31].

\subsection{Panel Design and $k$-Fold Cross Validation}

Stepwise logistic regression using forward selection and backward elimination was used to derive miRNA panels for (i) cirrhosis (F4) vs. mild disease (F0-2), and (ii) HCC vs. cirrhosis (F4). Pairwise correlations between individual miRNAs were performed to exclude miRNA combinations that displayed significant co-linearity within the model. K-fold cross-validation (5-fold) was used to assess the performance of the selected miRNA panels. The detailed panel design and k-fold cross validation methodology are described in Supplementary Methods.

\section{Conclusions}

This study identified the differential expression of several circulating miRNAs across a clinically-relevant spectrum of disease severity due to $\mathrm{CHC}$. Combining the expression levels of different miRNAs allows the optimisation of diagnostic panels that could potentially be developed to stratify $\mathrm{CHC}$ and diagnose early HCC. Prospective external validation of our miRNA panels is required, but these exciting preliminary results support the potential use of miRNAs as non-invasive biomarkers in the clinical assessment of $\mathrm{CHC}$ patients. We also provide further evidence that a miRNA panel could ultimately replace AFP in clinical practice as the non-invasive biomarker of choice in HCC.

\section{Patents}

A Complete Patent application (Australia and US only) was filed, entitled 'DETECTION OF LIVER DISEASE' (AU2018202716; Inventors Anna Weis, Richard Skoien, Grant Ramm). 
Supplementary Materials: Supplementary Materials can be found at http:/ /www.mdpi.com/1422-0067/20/4/ $864 /$ s1.

Author Contributions: R.S. and G.A.R. designed the current study, obtained funding and provided supervision. A.W. conducted the experiments and analysed the data. The advanced statistical analysis was performed by L.M. Patients were enrolled and clinical data were collected by R.S. and A.W. The manuscript was prepared by A.W. with support from D.A.C. and B.G., who also provided technical advice. All authors provided critical feedback, edited the manuscript and approved the final version of this manuscript.

Funding: This work was performed with financial support from the Cromwell Property Group Foundation administered via the Australian Liver Foundation, and the Royal Brisbane and Women's Hospital Foundation. Professor Grant A. Ramm is supported by a Senior Research Fellowship from the NHMRC of Australia (Grant No. APP1061332). Anna Weis was supported by a UQ International Scholarship and a QIMR Berghofer "top-up" scholarship. Berit Genz was supported by a Research Fellowship from German Research Foundation DFG, Bonn-Bad Godesberg, Germany (GE 2783/1-1). Diego A. Calvopina was sponsored by The Australian Liver Foundation (ALF) Pitcher Partners PhD Scholarship.

Acknowledgments: We kindly thank Leesa Wockner, David Smith, Lachlan Webb (QIMR Berghofer Statistics Unit, QIMR Berghofer, Brisbane, Australia) and Isabell Hoffmann (Institute of Medical Biostatistics, Epidemiology and Informatics, University Medical Centre, Johannes Gutenberg University of Mainz, Mainz, Germany) for statistical support and advice. We also thank Olivia Cullen, Deborah McIntyre and Karen Beckman (Department of Gastroenterology and Hepatology, Royal Brisbane and Women's Hospital, Brisbane, Australia) for their assistance during patient enrolment.

Conflicts of Interest: The authors declare no conflict of interest.

\section{Abbreviations}

$\begin{array}{ll}\text { AFP } & \text { Alpha-fetoprotein } \\ \text { ALT } & \text { Alanine aminotransferase } \\ \text { APRI } & \text { Aspartate aminotransferase to platelet ratio } \\ \text { AST } & \text { Aspartate aminotransferase } \\ \text { AUC } & \text { Area under the curve } \\ \text { CHC } & \text { Chronic Hepatitis C } \\ \text { 95\% CI } & \text { 95\% Confidence interval } \\ \text { DAA } & \text { Direct acting antivirals } \\ \text { FAK } & \text { Focal adhesion kinase } \\ \text { FIB-4 } & \text { Fibrosis-4 } \\ \text { HCC } & \text { Hepatocellular carcinoma } \\ \text { IQR } & \text { Interquartile range } \\ \text { miRNA } & \text { MicroRNA } \\ \text { NEK2 } & \text { NIMA-related kinase 2 } \\ \text { NIMA } & \text { Never in mitosis gene a } \\ \text { NPV } & \text { Negative predictive value } \\ \text { PIK3R1 } & \text { Phosphoinositide-3-Kinase Regulatory Subunit 1 } \\ \text { PPV } & \text { Positive predictive value } \\ \text { qRT-PCR } & \text { Real-time quantitative reverse transcription PCR } \\ \text { ROC } & \text { Receiver operating characteristic } \\ \text { SVR } & \text { Sustained virologic response }\end{array}$

\section{References}

1. Valery, P.C.; Laversanne, M.; Clark, P.J.; Petrick, J.L.; McGlynn, K.A.; Bray, F. Projections of primary liver cancer to 2030 in 30 countries worldwide. Hepatology 2018, 67, 600-611. [CrossRef] [PubMed]

2. Pawlotsky, J.M.; Negro, F.; Aghemo, A.; Berengue, M.; Dalgard, O.; Dusheiko, G.; Marra, F.; Puoti, M.; Wedemeyer, H. EASL Recommendations on Treatment of Hepatitis C 2018. J. Hepatol. 2018, 69, 461-511. [CrossRef] [PubMed]

3. Wedemeyer, H.; Dore, G.J.; Ward, J.W. Estimates on HCV disease burden worldwide-filling the gaps. J. Viral Hepat 2015, 22, 1-5. [CrossRef] [PubMed] 
4. $\quad$ Foster, G.R.; Irving, W.L.; Cheung, M.C.; Walker, A.J.; Hudson, B.E.; Verma, S.; McLauchlan, J.; Mutimer, D.J.; Brown, A.; Gelson, W.T.; et al. Impact of direct acting antiviral therapy in patients with chronic hepatitis C and decompensated cirrhosis. J. Hepatol. 2016, 64, 1224-1231. [CrossRef] [PubMed]

5. Verveer, C.; Zondervan, P.E.; ten Kate, F.J.; Hansen, B.E.; Janssen, H.L.; de Knegt, R.J. Evaluation of transient elastography for fibrosis assessment compared with large biopsies in chronic hepatitis B and C. Liver Int. 2012, 32, 622-628. [CrossRef] [PubMed]

6. Lin, Z.H.; Xin, Y.N.; Dong, Q.J.; Wang, Q.; Jiang, X.J.; Zhan, S.H.; Sun, Y.; Xuan, S.Y. Performance of the aspartate aminotransferase-to-platelet ratio index for the staging of hepatitis C-related fibrosis: An updated meta-analysis. Hepatology 2011, 53, 726-736. [CrossRef] [PubMed]

7. Vallet-Pichard, A.; Mallet, V.; Nalpas, B.; Verkarre, V.; Nalpas, A.; Dhalluin-Venier, V.; Fontaine, H.; Pol, S. FIB-4: An inexpensive and accurate marker of fibrosis in HCV infection. comparison with liver biopsy and fibrotest. Hepatology 2007, 46, 32-36. [CrossRef] [PubMed]

8. Singal, A.; Volk, M.L.; Waljee, A.; Salgia, R.; Higgins, P.; Rogers, M.A.; Marrero, J.A. Meta-analysis: Surveillance with ultrasound for early-stage hepatocellular carcinoma in patients with cirrhosis. Aliment. Pharmacol. Ther. 2009, 30, 37-47. [CrossRef]

9. Reid, G.; Kirschner, M.B.; van Zandwijk, N. Circulating microRNAs: Association with disease and potential use as biomarkers. Crit. Rev. Oncol. Hematol. 2011, 80, 193-208. [CrossRef]

10. Cermelli, S.; Ruggieri, A.; Marrero, J.A.; Ioannou, G.N.; Beretta, L. Circulating microRNAs in patients with chronic hepatitis C and non-alcoholic fatty liver disease. PLoS ONE 2011, 6, e23937. [CrossRef]

11. Bihrer, V.; Friedrich-Rust, M.; Kronenberger, B.; Forestier, N.; Haupenthal, J.; Shi, Y.; Peveling-Oberhag, J.; Radeke, H.H.; Sarrazin, C.; Herrmann, E.; et al. Serum miR-122 as a biomarker of necroinflammation in patients with chronic hepatitis C virus infection. Am. J. Gastroenterol. 2011, 106, 1663-1669. [CrossRef] [PubMed]

12. Bruix, J.; Sherman, M.; American Association for the Study of Liver, D. Management of hepatocellular carcinoma: An update. Hepatology 2011, 53, 1020-1022. [CrossRef] [PubMed]

13. Angileri, F.; Morrow, G.; Scoazec, J.Y.; Gadot, N.; Roy, V.; Huang, S.; Wu, T.; Tanguay, R.M. Identification of circulating microRNAs during the liver neoplastic process in a murine model of hereditary tyrosinemia type 1. Sci. Rep. 2016, 6, 27464. [CrossRef] [PubMed]

14. Josson, S.; Gururajan, M.; Hu, P.; Shao, C.; Chu, G.Y.; Zhau, H.E.; Liu, C.; Lao, K.; Lu, C.L.; Lu, Y.T.; et al. miR-409-3p/-5p promotes tumorigenesis, epithelial-to-mesenchymal transition, and bone metastasis of human prostate cancer. Clin. Cancer Res. 2014, 20, 4636-4646. [CrossRef] [PubMed]

15. Zhang, G.; Liu, Z.; Xu, H.; Yang, Q. miR-409-3p suppresses breast cancer cell growth and invasion by targeting Akt1. Biochem. Biophys. Res. Commun. 2016, 469, 189-195. [CrossRef] [PubMed]

16. Hou, J.; Lin, L.; Zhou, W.; Wang, Z.; Ding, G.; Dong, Q.; Qin, L.; Wu, X.; Zheng, Y.; Yang, Y.; et al. Identification of miRNomes in human liver and hepatocellular carcinoma reveals miR-199a/b-3p as therapeutic target for hepatocellular carcinoma. Cancer Cell 2011, 19, 232-243. [CrossRef] [PubMed]

17. Bala, S.; Tilahun, Y.; Taha, O.; Alao, H.; Kodys, K.; Catalano, D.; Szabo, G. Increased microRNA-155 expression in the serum and peripheral monocytes in chronic HCV infection. J. Transl. Med. 2012, 10, 151. [CrossRef]

18. Koberle, V.; Kronenberger, B.; Pleli, T.; Trojan, J.; Imelmann, E.; Peveling-Oberhag, J.; Welker, M.W.; Elhendawy, M.; Zeuzem, S.; Piiper, A.; et al. Serum microRNA-1 and microRNA-122 are prognostic markers in patients with hepatocellular carcinoma. Eur. J. Cancer 2013, 49, 3442-3449. [CrossRef]

19. Coulouarn, C.; Factor, V.M.; Andersen, J.B.; Durkin, M.E.; Thorgeirsson, S.S. Loss of miR-122 expression in liver cancer correlates with suppression of the hepatic phenotype and gain of metastatic properties. Oncogene 2009, 28, 3526-3536. [CrossRef]

20. Varnholt, H.; Drebber, U.; Schulze, F.; Wedemeyer, I.; Schirmacher, P.; Dienes, H.P.; Odenthal, M. MicroRNA gene expression profile of hepatitis C virus-associated hepatocellular carcinoma. Hepatology 2008, 47, 1223-1232. [CrossRef]

21. Sun, H.; Cui, C.; Xiao, F.; Wang, H.; Xu, J.; Shi, X.; Yang, Y.; Zhang, Q.; Zheng, X.; Yang, X.; et al. miR-486 regulates metastasis and chemosensitivity in hepatocellular carcinoma by targeting CLDN10 and CITRON. Hepatol. Res. 2015, 45, 1312-1322. [CrossRef]

22. Wang, L.; Liu, M.; Zhu, H.; Rong, W.; Wu, F.; An, S.; Liu, F.; Feng, L.; Wu, J.; Xu, N. Identification of recurrence-related serum microRNAs in hepatocellular carcinoma following hepatectomy. Cancer Biol. Ther. 2015, 16, 1445-1452. [CrossRef] 
23. Huang, X.P.; Hou, J.; Shen, X.Y.; Huang, C.Y.; Zhang, X.H.; Xie, Y.A.; Luo, X.L. MicroRNA-486-5p, which is downregulated in hepatocellular carcinoma, suppresses tumor growth by targeting PIK3R1. FEBS J. 2015, 282, 579-594. [CrossRef]

24. Fu, S.J.; Chen, J.; Ji, F.; Ju, W.Q.; Zhao, Q.; Chen, M.G.; Guo, Z.Y.; Wu, L.W.; Ma, Y.; Wang, D.P.; et al. MiR-486-5p negatively regulates oncogenic NEK2 in hepatocellular carcinoma. Oncotarget 2017, 8, 52948-52959. [CrossRef]

25. Ding, J.; Huang, S.; Wu, S.; Zhao, Y.; Liang, L.; Yan, M.; Ge, C.; Yao, J.; Chen, T.; Wan, D.; et al. Gain of miR-151 on chromosome 8q24.3 facilitates tumour cell migration and spreading through downregulating RhoGDIA. Nat. Cell Biol. 2010, 12, 390-399. [CrossRef]

26. Okajima, W.; Komatsu, S.; Ichikawa, D.; Miyamae, M.; Kawaguchi, T.; Hirajima, S.; Ohashi, T.; Imamura, T.; Kiuchi, J.; Arita, T.; et al. Circulating microRNA profiles in plasma: Identification of miR-224 as a novel diagnostic biomarker in hepatocellular carcinoma independent of hepatic function. Oncotarget 2016, 7, 53820-53836. [CrossRef]

27. Liu, A.M.; Zhang, C.; Burchard, J.; Fan, S.T.; Wong, K.F.; Dai, H.; Poon, R.T.; Luk, J.M. Global regulation on microRNA in hepatitis B virus-associated hepatocellular carcinoma. OMICS 2011, 15, 187-191. [CrossRef]

28. Guo, L.; Li, W.; Hu, L.; Zhou, H.; Zheng, L.; Yu, L.; Liang, W. Diagnostic value of circulating microRNAs for liver cirrhosis: A meta-analysis. Oncotarget 2018, 9, 5397-5405. [CrossRef]

29. Ding, Y.; Yan, J.L.; Fang, A.N.; Zhou, W.F.; Huang, L. Circulating miRNAs as novel diagnostic biomarkers in hepatocellular carcinoma detection: A meta-analysis based on 24 articles. Oncotarget 2017, 8, 66402-66413. [CrossRef]

30. Huang, Y.H.; Liang, K.H.; Chien, R.N.; Hu, T.H.; Lin, K.H.; Hsu, C.W.; Lin, C.L.; Pan, T.L.; Ke, P.Y.; Yeh, C.T. A Circulating MicroRNA Signature Capable of Assessing the Risk of Hepatocellular Carcinoma in Cirrhotic Patients. Sci. Rep. 2017, 7, 523. [CrossRef]

31. Team, R.C. R: A Language and Environment for Statistical Computing; R Foundation for Statistical Computing: Vienna, Austria, 2013; ISBN 3-900051-07-0. 